Revue d'histoire de l'enfance « irrégulière »

Le Temps de l'histoire

2 | 1999

Cent ans de répressions des violences à enfants

\title{
René Bérenger et la loi de 1898
}

Jacques Bourquin

\section{OpenEdition}

Journals

Édition électronique

URL : http://journals.openedition.org/rhei/31

DOI : $10.4000 /$ rhei.31

ISBN : 978-2-7535-1639-7

ISSN : 1777-540X

Éditeur

Presses universitaires de Rennes

Édition imprimée

Date de publication : 15 novembre 1999

Pagination : 59-68

ISSN : 1287-2431

Référence électronique

Jacques Bourquin, «René Bérenger et la loi de 1898 », Revue d'histoire de l'enfance « irrégulière » [En ligne], 2 | 1999, mis en ligne le 30 juillet 2010, consulté le 04 décembre 2020. URL : http:// journals.openedition.org/rhei/31 ; DOI : https://doi.org/10.4000/rhei.31

(c) PUR 


\section{René Bérenger et la loi de 1898}

\section{Jacques Bourquin $^{(1)}$}

(1) Président de

l'Association pour

l'histoire de l'éducation surveillée et de la protection judiciaire des mineurs (AHES-PJM).

(2) L'Assiette au beurre, $n^{\circ} 463,12 / 02 / 1910$, illustré par Radiguet (le père de l'écrivain

Raymond Radiguet).

(3) Bernard SCHNAP-

PER, "Le sénateur

Bérenger et les progrès de la répression pénale en France, 1870-1914”, Annales de la Faculté de droit d'Istambul, $\mathrm{n}^{\circ} 48$, 1979, repris dans Voies nouvelles en bistoire $d u$ droit, la justice, la famille, la répression pénale (XVIeXXe siècles), Paris, PUF, 1991, p. $355-373$. magistrat, futur fondateur de la Colonie de Mettray, évoquait, en parlant des enfants vagabonds des villes qu'il envoyait à la maison d'éducation correctionnelle de la Petite Roquette, les "innocents coupables". 
(4) Arnould BONNEVILLE de MARSANGY, Moralisation de l'enfance coupable, Paris, 1867, p. 5.

(5) Revue pénitentiaire, 1915, p. 675.
Un peu plus tard, en 1867, Arnould Bonneville de Marsangy associait aussi l'image de l'enfant victime à celle de l'enfant coupable : "Cette enfance misérable, ignorante, abandonnée, qui tôt ou tard constituera la classe entière des malfaiteurs $\gg .{ }^{(4)}$

En 1875, René Bérenger et Théophile Roussel reviennent sur cette idée qui lie l'enfance victime à l'enfance coupable : "C'est parmi les enfants victimes que l'on retrouve les enfants coupables, c'est en intervenant auprès des enfants victimes que l'on prévient la délinquance juvénile ».

René Bérenger, magistrat, député à l'Assemblée nationale en 1871, éphémère ministre des Travaux publics en 1873, puis sénateur inamovible de 1876 jusqu'à sa mort, était fils de magistrat. Son père, Alphonse Bérenger de la Drôme, membre de la Cour de cassation, Pair de France sous la Monarchie de Juillet, avait fondé avec Charles Lucas, un des introducteurs en France de la science pénitentiaire, et F.-A. Demetz, la Société de patronage des jeunes libérés de la Seine, œuvre dont le rôle était de suivre et d'accompagner les jeunes détenus à leur sortie de prison et de favoriser leur réadaptation dans la société.

René Bérenger est à ce titre un héritier; il s'inscrit pleinement dans la continuité de l'œuvre de son père en faveur des détenus et sera, à partir de 1877, président de la Société de patronage des libérés puis président de la Société générale des prisons. Il succéde à Charles Lucas à l'Institut et lui consacre une longue étude. Enfin, il poursuit l'œuvre de F.-A. Demetz à Mettray, dont il est administrateur à partir de 1879, puis président de 1909 à sa mort. Très lié à Théophile Roussel, René Bérenger sera vice-président du Conseil supérieur de l'Assistance publique. Très naturellement, son œuvre philanthropique devait l'amener à associer le sort des enfants victimes et des enfants coupables, et c'est dans cette voie que cet héritier est aussi un novateur.

Républicain convaincu, René Bérenger était aussi profondément catholique, rapprochement peu fréquent en ces débuts de la IIIe République, ce qui dénote chez lui une indépendance d'esprit certaine, que l'on retrouve en particulier chez certains membres fondateurs de la Société générale des prisons. Il exprimait dans un discours fait au Sénat, et qui fut repris dans un article que la Revue pénitentiaire ${ }^{(5)}$ lui 
consacrait au moment de sa mort, "Je suis un ferme républicain, tout en étant un conservateur, et j'entends rester un ferme catholique ». Martine Kaluszynski, qui a consacré un travail aux hommes de la Société générale des prisons, évoque les fondateurs que sont Dufaure, Picot, Roussel, Voisin, Bérenger qui unissent leur adhésion à la foi chrétienne et à la République, comme les porteurs d'un devoir social qui passe par une restauration de l'ordre moral liée à des valeurs religieuses : "ils ont une conception de l'ordre social qui attache du prix à la religion en tant que base de l'organisation sociale ". ${ }^{(6)}$ Ils s'inscrivent dans ce courant du catholicisme social qui s'exprime et qui est légitimé par Rome dans l'encyclique Rerum novarum. Il y a chez eux, comme chez leurs prédécesseurs de la Société royale des prisons sous la Restauration, une sorte de filiation entre le pénal et le social. Ils proposent à la République des politiques de prévention qui s'articulent autour de la religion, de la morale, de la famille.

Dès 1874, René Bérenger avait appuyé à la Chambre, puis au Sénat, les premières lois dont Théophile Roussel avait été le promoteur, en particulier celle du 19 mai 1874 sur le travail des enfants et leur emploi dans les exercices périlleux. En tant que président de la Société générale des prisons, il avait contribué à l'élaboration d'un projet de loi sur la protection des enfants abandonnés et maltraités, première grande loi d'assistance à l'enfance, déposée par Théophile Roussel en 1881 et qui ne sera votée, après de multiples projets successifs, que le 24 juillet 1889 .

La loi de 1898, soutenue par René Bérenger, souligne le lien entre l'enfant coupable et l'enfant victime. Dans le livre qu'il a consacré à l'histoire de la conceptualisation de la déviance juvénile, Jean-Marie Renouard note "l'utilisation de la déviance du second pour pointer celle de sa famille. En passant du coupable à l'enfant victime, on passe de la responsabilité individuelle à la responsabilité familiale. On déculpabilise en partie l'enfant pour mieux culpabiliser totalement sa famille ". ${ }^{(7)}$ Nous ne citons que pour mémoire les autres lois dont René Bérenger est l'instigateur, celles sur la libération conditionnelle, sur le sursis, sur la relégation. Il s'inscrit alors pleinement dans la lignée de Raymond Saleilles et du mouvement de la défense sociale, et s'affirme dans le domaine pénal et pénitentiaire comme un défenseur de l'individualisation de la peine.
(6) Martine KALUS-

ZYNSKI, Les Hommes de la Société générale des prisons (1877-1900), CERAF, 1993, n.p.

(7) Jean-Marie RENOUARD, De l'enfant coupable à l'enfant inadapté, Ed. du Centurion, Paris, 1990, p. 62 . 
(8) Journal officiel,

Documents du Sénat, 2 mai $1898, n^{\circ} 69$, p. 101.
La loi du 19 avril 1898 complète la loi du 24 juillet 1889 ; elle introduit la voie pénale par rapport aux violences à enfants. Celle qui permet de punir les parents.

\section{LE RÔLE DE RENÉ BÉRENGER DANS LÉLABORATION DE LA LOI DU 19 AVRIL 1898}

\section{Le rapport de la commission sénatoriale}

On sait que, suite à quelques affaires qui, dans le courant des années 1890, émurent fortement l'opinion publique, plusieurs projets de loi pour protéger les enfants victimes de sévices sont déposés à la Chambre par Henry Cochin, Georges Berry, Julien Goujon et Odilon Barrot. M. de Folleville rend alors un rapport qui est adopté par la Chambre sans discussion, après déclaration d'urgence le 11 juin 1897. Ce projet de loi est transmis le 14 juin au Sénat (JO du 23/01/1898), qui désigne une commission dont le sénateur René Bérenger est le rapporteur. Ce dernier rendit son rapport le 1er avril 1898. L'ensemble des points retenus par la Chambre le furent aussi par la commission. Toutefois René Bérenger proposa quelques dispositions nouvelles ${ }^{(8)}$ :

- élever, en cas de violence, de 13 à 15 ans l'âge de la protection; il était de 11 ans avant l'intervention de la Chambre, qui l'avait porté à 13 ans;

- remplacer par «fait des blessures ou porté des coups » la formule « exercice de violences quelconques" préconisée par la Chambre, ce qui est un moyen de préciser la nature de l'infraction. René Bérenger souhaite cette modification, voulant éviter de «soulever la délicate question du droit de correction qui doit rester un des attributs incontestables de la puissance paternelle ou de ses délégations »;

- remplacer, en matière d'exposition et de délaissement, l'expression "enfants au-dessous de 7 ans accomplis " par la formulation "enfants hors d'état de se protéger par eux-mêmes ", beaucoup plus générale. "Ce n'est pas l'âge, précise René Bérenger, qui rend l'acte à la fois criminel et dangereux, c'est l'impossibilité où se trouve la victime de s'y soustraire ". Par cette formulation nouvelle, la protection s'étend donc aux enfants en général, et plus particulièrement aux incapables, "idiots, infirmes, aliénés que la loi doit protéger". 
Lors du débat au Sénat du 10 mars, ce point sera vivement contesté par le sénateur Monservin, ${ }^{(9)}$ qui considéra que ne pas fixer d'âge laissait au juge trop de latitude et qu'il y avait un risque de trop se référer à une situation au détriment d'une loi précise.

René Bérenger et la commission suivent totalement le projet de la Chambre, qui prévoit que l'instruction pourra pourvoir provisoirement à la garde de l'enfant en le confiant à un parent, à une personne charitable, à l'Assistance publique; mais pourquoi, ajoute René Bérenger, «ne pas autoriser la remise de l'enfant à une institution charitable, mieux à même de recueillir l'enfant que les particuliers ou l'Assistance publique".

René Bérenger apparaît là comme un des promoteurs des patronages, dont le rôle, dès 1889 , est de favoriser une pénétration pédagogique des familles, puis ultérieurement, et c'est là un des vœux de la loi de 1898, d'éviter pour les enfants coupables le recours au placement dans les colonies pénitentiaires et correctionnelles.

On verra combien le débat au Sénat sera marqué par une tentative de limiter les pouvoirs des patronages.

Le rapport de la commission aborde un dernier point, celui de la déchéance de la puissance paternelle. Elle n'était prononcée de plein droit que dans un petit nombre de cas. La commission propose qu'elle soit étendue à tous les faits prévus par le projet de loi, c'est-à-dire à un bien plus grand nombre de délits; en fait, le but est moins la privation de l'ensemble des droits que celle d'un seul : le droit de garde. "Les tribunaux, précise René Bérenger, pourront ainsi enlever le droit de garde aux parents et le confier à d'autres sans toucher autrement à la puissance paternelle». La proposition sera considérée à l'époque comme une des innovations importante de la loi de 1898.

On peut être étonné, à la lecture du rapport de René Bérenger, que ne figure pas l'élément qui sera au cœur du débat au Sénat : le droit pour les associations protectrices de l'enfance reconnues d'utilité publique, comme certains patronages, de poursuivre, par citation directe ou en se portant partie civile, les auteurs de violences et attentats commis sur les enfants. Cette proposition, qui constitue l'article $7 \mathrm{du}$ projet, aurait été introduite après la remise du rapport par Théophile 
(10) Séance extraordinaire du 09/02/1898, Bulletin des sociétés de patronage, 1898 , p. 21 et sq.
Roussel et Paul Strauss. Elle avait toutefois fait l'objet d'un débat le 9 février 1898 devant l'Union des sociétés de patronage, où René Bérenger avait présenté les travaux de la commission. ${ }^{(10)}$

Le rapport n'évoque pas non plus la modification introduite par René Bérenger dans l'article 4, où il associe la protection des enfants victimes et des enfants coupables. Cette modification, qui nous apparaît aujourd'hui comme un point fort du projet, n'amène aucune réaction au moment du débat et du vote. Est-elle passée inaperçue ou correspondait-elle tout simplement à une évidence pour les sénateurs? Il fallait, dans un but de prévention de la délinquance, associer l'enfant victime et l'enfant coupable, et les faire bénéficier des mêmes mesures. C’était, depuis de longues années, nous le savons, l'opinion de René Bérenger, de Théophile Roussel et des membres des comités de défense des enfants traduits en justice.

\section{Le débat au Sénat}

Il se déroule les 10, 21 et 22 mars 1898. Le texte sera définitivement voté le 5 avril.

L'ensemble des articles amendés par la commission sénatoriale est adopté sans discussion, à l'exception du point relatif à la fixation de l'âge soulevé par le sénateur Monservin, que nous avons évoqué précédemment.

Le débat ne prend vraiment forme qu'à partir de la discussion de l'article 7 relatif " aux droits de poursuite par citation directe ou en se portant partie civile qui pourraient être accordés aux associations protectrices de l'enfance". Cet article est défendu avec ténacité par René Bérenger et Paul Strauss, au cours des trois séances du Sénat : "Il ne faut pas se contenter de protéger l'enfance malheureuse et de multiplier les pénalités", ce qui était l'objet des premiers articles de la loi de 1898, "il faut faire connaître aux tribunaux les actes de cruauté, les sévices". Ils appuient leur argumentation sur les législations anglaises et américaines, où des sociétés de protection de l'enfance sont pourvues de ces pouvoirs. Évoquant des affaires récentes et médiatisées qui sont à l'origine du projet de loi, comme le supplice de la petite Borlet en 1892, ils font référence aux réticences, à la répulsion 
même qu'ont les voisins à dénoncer, à signaler. L'action publique n'est souvent suivie que trop tardivement.

Les réactions à ces arguments sont très vives; elles viennent autant des sénateurs de la gauche républicaine (Léonce de Sal, Leporché) que des conservateurs (H. de Saisy, de Chamaillard). Elles sont d'abord d'ordre juridique : " c'est une modification profonde de notre législation [...]. L'association peut déposer une plainte; elle ne peut saisir les tribunaux ». C'est surtout la défense de la famille qui est au cœur du débat : "inquisition intolérable "; " citoyens obligés de se murer chez eux pour empêcher les oreilles indiscrètes "; "pression intolérable sur le père, la mère "; " on fausse le libre arbitre "; " comme vous traitez mal la famille, vous les protecteurs de la famille "... (11)

L'Union française de sauvetage de l'enfance, un des premiers patronages, dont Théophile Roussel est le vice-président, est mise en cause, accusée de malversation. On laisse entendre les risques de récupération religieuse ou autres qu'il y aurait à accorder un tel pouvoir aux associations.

René Bérenger et Paul Strauss, qui font de cet article un élément majeur du rôle des patronages dans la prévention, nuancent leur propos en disant que, si les patronages "sont amenés à donner des avertissements aux parents, à les conseiller, la plainte ne sera que l'ultime recours". Ils soulignent l'importance que la Société générale des prisons et le Comité de défense des enfants traduits en justice, accordent au vote de cet article 7 . Le garde des sceaux Milliard apporte la conclusion du débat : " C'est quand un enfant a été maltraité et corrompu qu'il est prêt à toutes les mauvaises besognes [...]. Il est vrai de dire que les crimes et les délits commis contre les enfants préparent et engendrent les délits et crimes commis par les jeunes gens ". ${ }^{(12)}$ À nouveau enfants victimes et enfants coupables se rejoignent.

L'échec majeur du projet de loi, c'est le rejet de l'article 7 après trois jours de débat. 226 sénateurs votent contre et seulement 27 pour. On peut parler là d'une défaite relative de la néophilantropie, porteuse du projet des patronages et de la pénétration pédagogique des familles.

Ce qui est au cœur du débat, c'est bien le rôle et la place à accorder aux associations. Et le débat est déjà ancien. Pourtant, dans les années 1880, la République, très préoccupée de l'éducation et de la moralisa-
(11) J.O., débat parlementaire, Sénat, séances du 21 et 22 mars.

(12) J.O., débat parlementaire, Sénat, séance du 22 mars. 
(13) Georges LELOIR, "Etude sur la Loi du 19 Avril 1898", in Journal des Parquets, Paris, Ed. Rousseau, 1903, p. 203-232.

(14) Paul JOLLY, Revue Pénitentiaire, 1903, p. 337 et suivantes. tion de l'enfant, va se servir des associations pour lutter contre la gestion pénale des mineurs organisée par l'Administration pénitentiaire, cette dernière se situant trop dans la continuité du Second Empire. L'appel aux associations est un moyen, dans ce domaine, de substituer la moralisation de l'enfant "coupable" à la répression. Les associations, investies de l'intervention auprès des enfants victimes, se verront confier des droits de puissance paternelle, de défense des mineurs, de tutelle légale...

C'est dans cette continuité que le législateur de 1898 propose le droit de poursuite pour les associations. Le pouvoir accordé à une association, c'était élargir le champ de liberté réservé jusqu'alors au citoyen, c'était le risque de déléguer la vindicte publique à des particuliers et, derrière cela, le risque de donner trop de pouvoir aux congrégations, voire aux syndicats. Le sujet était très sensible. La proposition arrivait bien avant son temps.

La loi est définitivement adoptée par la Chambre, le 19 avril 1898, sans débat.

\section{POUR CONCLURE}

Les réactions à la loi apparurent rapidement, mettant en question cependant non plus l'article 7 et les pouvoirs des patronages, mais l'article 4 qui évoquait "les crimes et délits commis sur et par les enfants » et qui n'avait fait l'objet d'aucun débat au Sénat. Les incidences de cet article furent très vite discutées. Georges Leloir ${ }^{(13)}$ signale dès 1899 que, "en assimilant les auteurs d'infraction aux enfants victimes, la loi de 1898 permettait au magistrat d'éviter pour l'enfant coupable auteur de délits la détention préventive ou l'envoi en colonie pénitentiaire ", ce qui était de toute évidence le souhait des promoteurs des patronages. Très vite le pas à franchir parut excessif et Paul Jolly, ${ }^{(14)}$ juge d'instruction à Paris, écrivait en 1903 que, "pour René Bérenger, l'idée d'éducation primait celle de répression, même pour les enfants coupables". Mais, poursuivait-il, "cette révolution du législateur n'a pas eu d'effets ». Pour illustrer son propos, Paul Jolly donne les statis- 
tiques du tribunal de la Seine en matière de mineurs, pour la période qui va de juin 1898 à décembre 1901 :

3898 mineurs poursuivis

1371 renvoyés devant le tribunal

- 67 acquittés simples

- 149 condamnés comme discernants

- 1155 acquittés comme non discernants

parmi ces derniers :

- 213 sont remis à leurs parents

- 940 sont envoyés en correction

- 2 sont placés en application de la loi de 1898 (placements en patronage).

Était-ce la faillite de la loi, qui confondait deux situations différentes, celle d'enfant coupable et celle d'enfant victime? C'était apparemment la faillite de la reconnaissance des patronages et de la loi de 1898, qui n'avait pas prévu d'intervention financière pour la garde des enfants.

Les choses sont peut-être un peu plus complexes. Lors de son bureau du 17 novembre 1902, l'Union des sociétés de patronage et des institutions préventives adresse à tous les membres de la magistrature une circulaire signée par les "présidents d'ouvre et les criminalistes les plus éminents ", dont Théophile Roussel, René Bérenger, G. d'Haussonville, Louis Albanel... : "Il est à notre avis, écrivent-ils, très préjudiciable aux intérêts de l'enfant que l'envoi dans une colonie pénitentiaire ne soit pas plus souvent prononcé [...]. Il va de soi que nous n'oublions pas les mesures nouvelles (remise de l'enfant à une personne ou à une institution charitable ou à l'Assistance Publique) que la loi de 1898 met à la disposition des magistrats [...]. L'envoi en correction préserve l'enfant des chutes successives qui en font de très bonne heure un professionnel du délit [...]. Il ne faut pas oublier que l'envoi d'un enfant dans une colonie pénitentiaire n'est qu'une mesure d'éducation et pas une peine [...]. La maison de correction n'est pas, comme on se l'imagine trop facilement, une sorte de bagne pour enfants $»{ }^{(15)}$

Assiste-t-on à un revirement des promoteurs de la loi de 1898 ? Pas exactement, car la solution qui est proposée par la circulaire consiste en "une combinaison de l'envoi en correction et du patronage. [...] L'enfant en cor-

(15) Revue Pénitentiaire, 1903, pp. 86-87. 
(16) Léonce ANDRÉ,

Les Tribunaux pour enfants et la Liberté Surveillée, Paris, 1914, p. 44. rection reste détenu quelques semaines avant d'être dirigé vers une colonie pénitentiaire. À ce moment, une société de patronage peut intervenir et demander que l'enfant lui soit remis [...] ; ainsi cet enfant envoyé à la correction jusqu'à 20 ans sera remis à la société de patronage avant même d'être entré à la maison de correction, qu'il ne connaîtra jamais s'il se conduit bien".

"Il est indispensable, concluent les rédacteurs de la circulaire, qu'un jugement prononçant l'envoi en maison de correction soit rendu avant la remise à une société de patronage ". Ceci peut relativiser les statistiques évoquées par Georges Leloir. On mentionne la décision de correction et on ne mentionne pas le placement en patronage qui peut suivre. L'enfant sera donc ainsi toujours sous la menace d'un éventuel recours à l'établissement de correction : "Il faut que l'enfant sache bien que, s'il manque à sa promesse, s'il ne suit pas les conseils qui lui sont prodigués, la société de patronage pourra l'envoyer en correction ".

En 1904, le mouvement s'inversait, ce n'était plus les enfants coupables qui pouvaient relever des patronages, c'était les colonies pénitentiaires qui étaient habilitées à recevoir les enfants victimes, en application de la loi du 28 juin sur les pupilles difficiles ou vicieux de l'Assistance publique. La confusion entre enfant victime et enfant coupable ne faisait que s'accentuer. Étaient-ils plus victimes que coupables ou l'inverse? Participant aux travaux préparatoires de la loi de 1912 sur les tribunaux pour enfants, René Bérenger demandait, à la séance du 31 janvier 1911, ${ }^{(16)}$ la suppression de la détention préventive pour les mineurs : "Le jour où un enfant est incarcéré, il sera à peu près perdu ».

C'est la loi de 1912 qui donna leur véritable légitimité aux patronages, ce qui, par voie de conséquence, entraîna dès l'après-guerre le déclin des colonies pénitentiaires. 